\title{
Intraoperative magnetic resonance imaging in pediatric neurosurgery: safety and utility
}

\author{
Mario Giordano, MD, ${ }^{1}$ Amir Samii, MD, PhD, ${ }^{1,2}$ Anna C. Lawson McLean, MD, ${ }^{1}$ \\ Helmut Bertalanffy, MD, PhD, ${ }^{1}$ Rudolf Fahlbusch, MD, PhD, ${ }^{1}$ Madjid Samii, MD, PhD, ${ }^{1}$ and \\ Concezio Di Rocco, MD'

\begin{abstract}
'Department of Neurosurgery, International Neuroscience Institute, Hannover; and 'Leibniz Institute for Neurobiology, Magdeburg, Germany
\end{abstract}

OBJECTIVE The use of high-field intraoperative MRI has been largely studied for the treatment of intracranial tumors in adult patients. In this study, the authors investigated the safety, advantages, and limitations of high-field iMRI for cranial neurosurgical procedures in pediatric patients, with particular attention to craniopharyngiomas and gliomas.

METHODS The authors performed 82 surgical procedures in patients under 16 years of age (range 0.8-15 years) over an 8-year period (2007-2014) using iMRI. The population was divided into 3 groups based on the condition treated: sellar region tumors (Group 1), gliomas (Group 2), and other pathological entities (Group 3). The patients' pre- and postoperative neurological status, the presence of residual tumor, the number of intraoperative scans, and complications were evaluated.

RESULTS In Group 1, gross-total resection (GTR) was performed in 22 (88\%) of the procedures and subtotal resection (STR) in 3 (12\%). In Group 2, GTR, STR, and partial resection (PR) were performed, respectively, in 15 (56\%), $7(26 \%)$, and $5(18 \%)$ of the procedures. In Group 3, GTR was performed in $28(93 \%)$ and STR in 2 (7\%) of the procedures. In cases of craniopharyngioma (Group 1) and glioma (Group 2) in which a complete removal was planned, iMRI allowed localization of residual lesions and attainment of the surgical goal through further resection, respectively, in $18 \%$ and $27 \%$ of the procedures. Moreover, in gliomas the resection could be extended from partial to subtotal in $50 \%$ of the cases. In $17 \%$ of the patients in Group 3, iMRI enabled the identification and further removal of tumor remnants. There was no intra- or postoperative complication related to the use of iMRI despite special technical difficulties in smaller children.

CONCLUSIONS In this study, the use of iMRI in children proved to be safe. It was most effective in increasing the extent of tumor resection, especially in patients with low-grade gliomas and craniopharyngiomas. The most prominent disadvantage of high-field iMRI was the limitation with respect to operative positioning due to the configuration of the surgical table.

http://thejns.org/doi/abs/10.3171/2016.8.PEDS15708

KEY WORDS intraoperative magnetic resonance imaging; pediatric; glioma; craniopharyngioma; oncology

$\mathrm{T}$ $\mathrm{HE}$ introduction of intraoperative magnetic resonance imaging (iMRI) has been an important innovation in the surgical treatment of intracranial tumors. The first reports of low-field iMRI date back to the mid-1990s..$^{14,19}$ In subsequent years, surgical protocols and MRI technology evolved, which allowed the introduction of high-field scanners for intraoperative imaging. Higher field strength is associated with higher signal-to-noise ratio and therefore better imaging quality. This development led to a wider range of potential applications with regard to both better accuracy in detecting the tumor and improved safety in its resection by delineating its spatial relationships with surrounding functionally relevant structures. Currently, iMRI is a well-established tool for safe and complete glioma resection in adults in combination with computer-based neuronavigation based on pre- and

ABBREVIATIONS DTI = diffusion tensor imaging; GTR = gross-total resection; iMRI = intraoperative MRI; MPRAGE = magnetization prepared rapid acquisition gradient echo; PR = partial resection; STR = subtotal resection.

SUBMITTED December 8, 2015. ACCEPTED August 5, 2016.

INCLUDE WHEN CITING Published online October 28, 2016; DOI: 10.3171/2016.8.PEDS15708. 
intraoperative images. ${ }^{6,11,18}$ Except for a few early preliminary reports, $, 12,13$ the use of iMRI in the pediatric population has only been investigated in a few recent studies..$^{1,5,21}$ In children, the extent of tumor removal is of paramount importance, as it is the main prognostic factor in malignant intracranial tumors, ${ }^{17,20}$ complete removal may be curative in benign intracranial neoplasms, and postoperative demonstration of unexpected tumor remnants may lead to reoperation. In this report, we present our experience with high-field iMRI in the pediatric population. We discuss the safety and utility of this technique and present shortterm patient outcomes.

\section{Methods \\ Patients}

Among all the patients treated in our institution using iMRI, all the children under 16 years old were considered for the present study, forming a series of 75 patients who underwent 82 procedures. All procedures were conducted between May 2007 and November 2014. The median age at the time of the operation was 7 years (range 0.8-15 years). Patient records were evaluated with regard to surgical aspects (planned percentage of resection, approach, grade of resection), intraoperative imaging, patient history and neurological status on admission and discharge, and postoperative complications.

We categorized the cases into 4 groups based on lesion histology and location (Table 1). Group 1 comprised 18 patients who underwent a total of 25 operations for treatment of sellar region tumors. Group 2A comprised 19 patients who each underwent 1 operation for the treatment of supratentorial gliomas. Group 2B comprised 8 patients who each underwent 1 operation for the treatment of infratentorial gliomas. Group 3 comprised 30 patients who each underwent 1 operation for the treatment of other histological entities.

\section{Operating Theater Setup}

All procedures were performed in a radiofrequencyshielded operating theater equipped with a high-field 1.5T MR scanner (Magnetom Espree, Siemens). The scanner has an integrated rotating table that allows the patient's head to be placed outside the 5-G line during the operation. Standard surgical instruments may be used outside this line without influencing the magnetic field. The surgical microscope (OPMI Pentero, Carl Zeiss) is fixed to the ceiling outside the 5-G line. The MRI unit is equipped with a neuronavigation system (Vector Vision Sky Navigation System, Brainlab). A glass wall separates the operating theater from the computer control room that is used to set up the scanner sequences, visualize the intraoperative images, and plan the surgery with the frameless navigation system. For intraoperative image acquisition, the surgical site is prepared as previously described ${ }^{7}$ and the table is rotated into the scanning position. Two headrest coils are available: a fixed coil with built-in supports for skull pins and a flexible coil that can be used for transsphenoidal surgery. These coils are standard and not specially designed for children.
TABLE 1. The 3 main groups of patients treated using intraoperative MRI

\begin{tabular}{lc}
\hline Patient Group and Tumor Type & No. of Surgical Procedures \\
\hline Group 1: sellar region tumors & \\
\hline Craniopharyngioma & 22 \\
\hline Rathke's cleft cyst & 2 \\
\hline Pituitary macroadenoma & 1 \\
\hline Group 2: gliomas & \\
\hline Group 2A: supratentorial & 9 \\
\hline Pilocytic astrocytoma (Gr I) & 3 \\
\hline Diffuse astrocytoma (Gr II) & 3 \\
\hline Glioblastoma (Gr IV) & 1 \\
\hline Anaplastic astrocytoma (Gr III) & 1 \\
\hline Oligodendroglioma (Gr II) & 1 \\
\hline Angiocentric glioma (Gr I) & 1 \\
\hline SEGA (Gr I) & 7 \\
\hline Group 2B: infratentorial & 1 \\
\hline Pilocytic astrocytoma (Gr I) & 2 \\
\hline Anaplastic astrocytoma (Gr III) & 3 \\
\hline Group 3: other pathologies & 3 \\
\hline Ependymoma (Gr II) & 3 \\
\hline Cortical dysplasia & 2 \\
\hline Ganglioglioma (Gr II) & \\
\hline Hamartoma & \\
\hline Germinoma & \\
\hline PNET & \\
\hline Others & \\
\hline
\end{tabular}

$\mathrm{Gr}=\mathrm{WHO}$ grade; $\mathrm{PNET}=$ primitive neuroectodermal tumor; $\mathrm{SEGA}=$ subepen dymal giant cell astrocytoma.

\section{Intraoperative Imaging}

MRI was performed before, during, and after surgery in each case. Before surgery, the patients underwent a complete MRI study, which was also used for the registration of intraoperative navigation. The standard protocol included the following sequences: T1-weighted magnetization-prepared rapid acquisition gradient echo (MPRAGE) (slice thickness $1 \mathrm{~mm}$, FOV $320 \mathrm{~mm}^{2}$, TE $3.5 \mathrm{msec}$, TR 2150 msec); T2-weighted (slice thickness $1 \mathrm{~mm}$, FOV $320 \mathrm{~mm}^{2}$, TE $505 \mathrm{msec}$, TR $3200 \mathrm{msec}$ ); fluid-attenuated inversion recovery (FLAIR) (slice thickness $1 \mathrm{~mm}$, FOV $320 \mathrm{~mm}^{2}$, TE $505 \mathrm{msec}$, TR $5000 \mathrm{msec}$ ); and diffusion tensor imaging (DTI) (slice thickness 3 mm, FOV $320 \mathrm{~mm}^{2}$, TE 107 msec, TR $3000 \mathrm{msec}$ ). Patients with contrast-enhancing lesions received gadopentetate dimeglumine $(0.2 \mathrm{ml} / \mathrm{kg}$, administered intravenously) before imaging.

For transsphenoidal surgeries performed without navigation, a flexible coil was used and the following sequences were acquired: T1-weighted coronal and sagittal spin echo sequences (slice thickness $3 \mathrm{~mm}$, FOV $230 \mathrm{~mm}^{2}$, TR $500 \mathrm{msec}$, TE $9.5 \mathrm{msec}$ ) and T2-weighted turbo spin echo sequences (slice thickness $3 \mathrm{~mm}$, FOV $230 \mathrm{~mm}^{2}$, TR 4570 msec, TE $120 \mathrm{msec}$ ). In cases of parasellar tumors, MR angiography was routinely performed. For intraoperative 
scans, the noncompatible nasal speculum was replaced with light nasal packing.

The images were evaluated and the tumor volume was segmented. For tumors not showing contrast enhancement, the area with signal abnormalities on FLAIR sequences was segmented. For tumors containing enhancing and nonenhancing areas, both parts were segmented.

For pre- and postoperative fiber tracking, the DTI data were transferred to the neuronavigation planning workstation (iPlan, Brainlab AG) for image processing using version 3.0 of the software. After rigid registration of the functional and anatomical sequences, the fiber tracking was performed. For the supine position, the DTI sequences were performed with separate settings from the anatomical images to avoid error in the direction analysis.

Intraoperative images were acquired after the surgeon stated that the surgical goal was reached, when there was suspicion of a tumor remnant that could be further removed, or to update the navigation system, for instance in patients with severe brain shift or an anatomical anomaly.

For intraoperative imaging, the same sequences as for preoperative imaging were used. In case of the surgeon's decision to proceed with further resection, the newly acquired images were used to identify residual tumor and to update the navigation system, compensating for the brainshift effect. Updated diffusion tensor images were used to reconstruct the fiber bundles and/or white matter tracts of interest.

\section{Results}

\section{Group 1: Sellar Region Tumors}

This group consisted of 15 patients with craniopharyngioma (22 procedures), 2 with Rathke's cleft cyst, and 1 with a pituitary macroadenoma (Table 1 ). The diagnoses of the 2 Rathke's cleft cysts were an unexpected intraoperative finding. The iMRI was used in these cases because the tentative preoperative diagnosis was craniopharyngioma. The duration of symptoms prior to surgery ranged from 1 to 18 months. The most common preoperative symptoms and signs were visual field deficit, hypophysealhypothalamic dysfunction, and intracranial hypertension, which occurred, respectively, in 10,9, and 7 cases.

Of the 22 procedures performed in patients affected by craniopharyngioma, 9 represented an initial operation and 13 were repeat operations for the treatment of recurrences. A transcranial (frontolateral or frontotemporal) route was used in 6 of the initial operations and 9 of the repeat operations, while a transsphenoidal route was chosen for the other 3 initial and 4 repeat operations.

Regarding the 9 patients who were undergoing a first operation for craniopharyngioma, a total removal of the lesion had been preoperatively planned and could be achieved in all cases. Complete removal was confirmed by the first iMRI in 8 of the 9 cases. In 1 case, the first iMRI revealed unexpected residual tumor at the pituitary stalk; the procedure was continued using updated navigation and complete removal of the lesion was confirmed by the second iMRI.

Regarding the 13 procedures for recurrence of craniopharyngioma, we were able to achieve complete removal in 10 cases ( 8 via a transcranial route and 2 via a transsphenoidal route) and subtotal resection in 3 cases (1 via a transcranial route and 2 via a transsphenoidal route). In this last group, complete resection was not planned preoperatively because of the expectation of adherence due to previous surgeries.

In 7 of the 10 cases in which complete resection was performed, it was confirmed by the initial iMRI study; in the other 3 cases, the initial iMRI study revealed residual tumor. In 1 patient there was a residual cystic part in the anterior part of the third ventricle; in another case a retrochiasmatic remnant was found (Fig. 1). In both cases, the second iMRI study confirmed complete resection. In the third case, 3 iMRI studies were necessary-due to the complex configuration of the only partially pneumatized sphenoid sinus in children under 5 years, an iMRI study was performed during the transsphenoidal approach to obtain a better orientation for drilling of the skull base.

In the 3 cases of recurrent craniopharyngioma in which only subtotal resection (STR) ( $\geq 90 \%$ of tumor volume) was performed, residual tumor was confirmed by iMRI to be strongly adherent to vascular structures (carotid artery, anterior cerebral artery, and posterior communicating artery). In one of these 3 cases the procedure was continued after the first imaging procedure because further removal of the mass was judged to be possible, and a second iMRI study confirmed reduction of the residual lesion.

We had no intra- or postoperative complication due to the use of iMRI. Ten patients experienced early postoperative electrolyte imbalance, which was corrected during their hospital stay. Two patients had CSF leakage that was treated with temporary lumbar drainage. Visual field deficit improved in 6 of the 7 patients with preoperative deficits and worsened in 1 patient; there were no visual field changes in the other cases.

The other 3 patients in this group ( 2 who had Rathke's cleft cysts and 1 with a pituitary adenoma) were all treated using a transsphenoidal approach. Only 1 iMRI session was needed, and it showed the complete evacuation-resection of the lesion in all 3 cases. No complication occurred.

The mean total time for preoperative MRI, including positioning, coil setting, and draping for Group 1, was 34 \pm 5 minutes; the corresponding mean total time for iMRI was $35 \pm 6$ minutes. In cases in which a flexible coil was used the mean time for iMRI was shorter, with a mean of 18 minutes of image acquisition and $5 \pm 3$ minutes for coil setting and draping.

\section{Group 2A: Supratentorial Gliomas}

Nineteen patients were treated for supratentorial gliomas (Table 1). The majority of these lesions (15 of 19) were low-grade tumors, and the most frequent histological diagnosis was pilocytic astrocytoma (9 cases). The highgrade lesions were glioblastomas ( 3 cases) and an anaplastic astrocytoma (1 case). The most common preoperative symptoms were intracranial hypertension (present in 16 patients) and seizures (occurring in 9 patients). Five patients had been previously surgically treated in other hospitals with partial resection or biopsy of the lesion.

The primary intention of surgery was gross-total resection (GTR) ( $\geq 98 \%$ of tumor volume) in 12 cases, STR $(\geq$ 

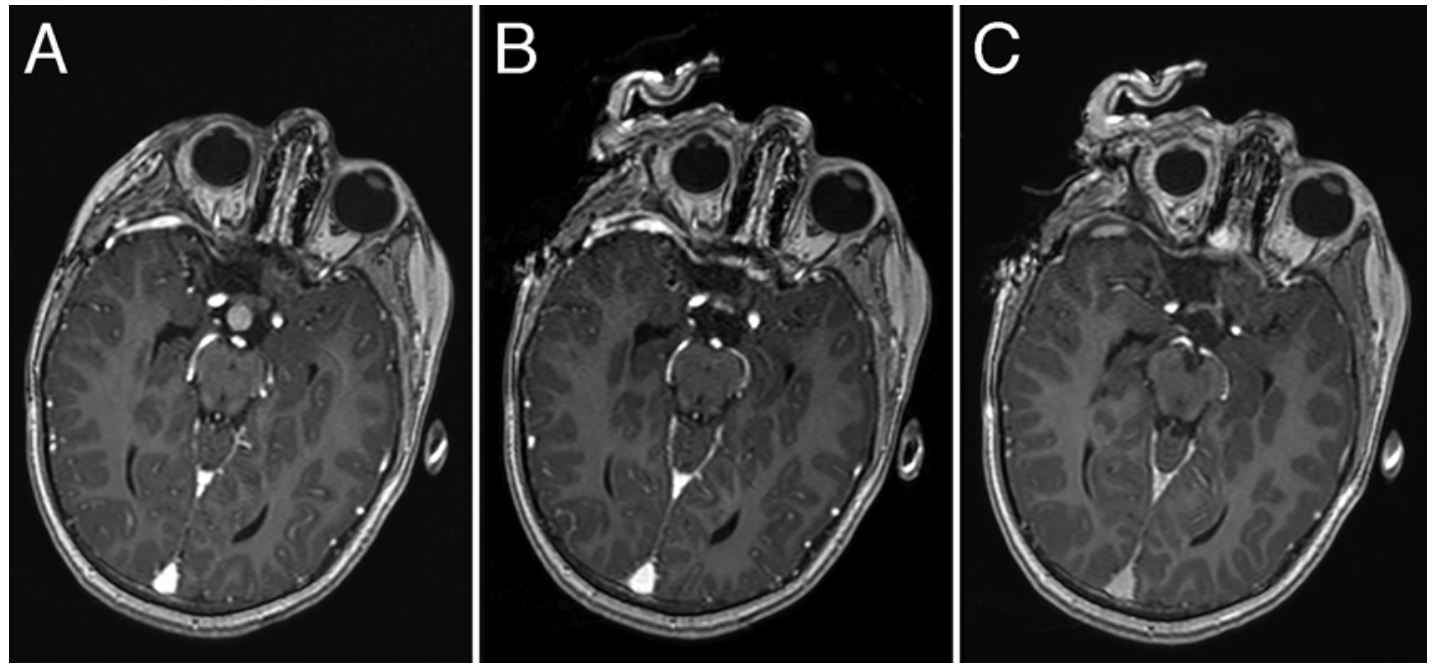

FIG. 1. Axial T1-weighted contrast-enhanced MR images obtained in a 6 -year-old child affected by craniopharyngioma. A: Preoperative image showing the lesion in contact with the optic chiasm. B: Image from first iMRI study, performed after resection via frontolateral approach, demonstrating a small remnant posterior to the optic chiasm. C: Image from second iMRI study showing complete removal of the lesion.

$90 \%$ of tumor volume) in 1 case, and partial resection (PR) ( $<90 \%$ of tumor volume) in 6 cases. Overall, at the end of the surgery, GTR was achieved in 12 cases, STR in 4, and $\mathrm{PR}$ in 3 .

In 9 of the 12 cases in which GTR was performed, only 1 iMRI procedure was necessary to confirm the intended extent of resection (Fig. 2). In the other 3 cases, GTR was verified at the second iMRI session. In these cases the surgeon judged that the preoperative goal had been reached before the iMRI, but images showed residual tumor, which was then removed with the help of updated navigation.

In 7 cases, either STR or PR was planned due to the tumor location and involvement of functional areas. In 4 cases (1 STR and 3 PR) only 1 iMRI session was necessary to verify the planned goal. In 3 of the 6 cases planned for PR, the use of iMRI allowed the degree of resection to be increased from PR to STR. In fact, after evaluation of the images the surgeon considered further tumor removal to be possible without damage to functional structures; for this reason, a second MRI study was performed and the extension of the resection was verified.

Intraoperative complications were rare and were not due to the use of iMRI. One patient experienced extensive intraoperative blood loss, and the procedure was stopped. Another patient developed an epidural hematoma, which was detected with iMRI and could be evacuated during the same surgery. Neurological status was improved in 6 patients and remained stable in 10. In 4 cases the patients demonstrated worsening of neurological status after the operation. In 3 of these 4 cases, the deterioration was only transient and improved during the hospital stay. In the remaining patient, hemiparesis was still present at discharge.

The mean total time for the preoperative MRI, including positioning, coil setting, and draping, was $39 \pm 6$ minutes. The corresponding mean total time for iMRI was 40 \pm 8 minutes.

\section{Group 2B: Infratentorial Gliomas}

Eight children in our study population were affected by infratentorial gliomas (Table 1). The tumors in this group were mainly low-grade tumors: 7 pilocytic astrocytomas and only 1 anaplastic astrocytoma. Preoperative neurological evaluation included signs of intracranial hypertension in 6 cases, coordination disturbances in 4, and cranial nerve deficit in 3 . Two patients had already been treated with PR in other facilities.

The primary intention of surgery included GTR in 3 cases, STR in 1, and PR in 4 (due to brainstem tumor location). The last iMRI demonstrated GTR in 3 cases, STR in 3 , and PR in 2.

In 2 of the 3 cases in which GTR was received, only 1 iMRI study was needed to confirm the achieved goal; in the other case 4 iMRI studies were needed. Without the use of iMRI, the surgeon would have interrupted the operation in this last case before the first iMRI procedure was performed. In fact, due to the critical location and the difficult microscopic identification of the lesion, several iMRI studies were performed to verify the presence and position of residual tumor. As in the previous group, we could increase the amount of tumor resection in some of the cases planned for PR: in 2 of the 4 cases an STR could be performed. These patients underwent 2 iMRI studies: after the first imaging procedure it was judged safe to continue the surgery with further removal. The mean total time for the preoperative MRI, including positioning, coil setting, and draping, was $49 \pm 5$ minutes. The corresponding mean total time for iMRI was $42 \pm 6$ minutes.

There were no intraoperative complications. Postoperatively, 1 patient had a urinary tract infection, and 1 patient had an infection of the central venous catheter. Seven patients showed no postoperative change in neurological condition. One patient had a transient worsening of preoperative ataxia. 

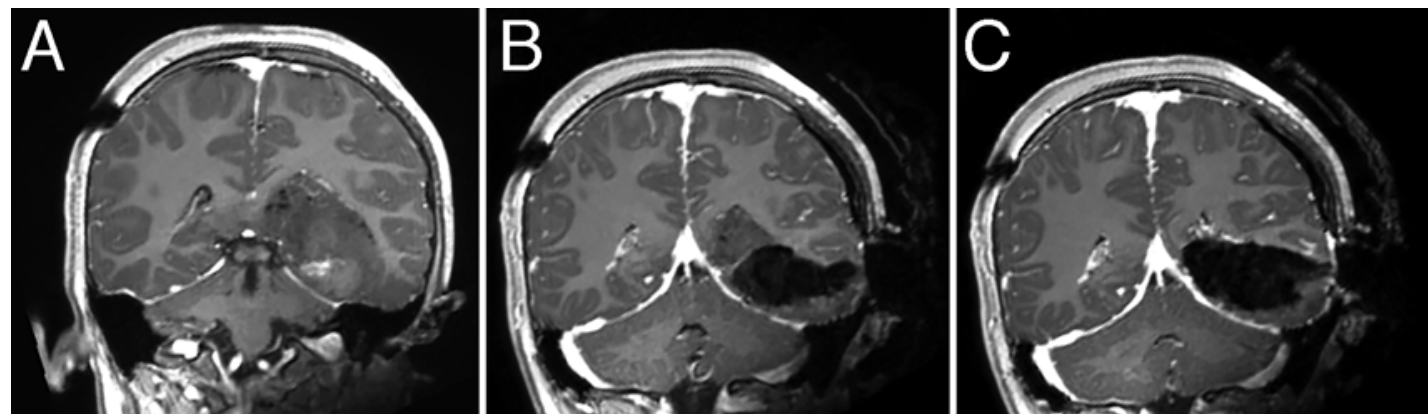

FIG. 2. Coronal T1-weighted contrast-enhanced MR images obtained in a 7-year-old child affected by a left temporo-parietal glioblastoma. A: Preoperative image. B: Image from first iMRI study visualizing a deep-seated residual tumor close to the left lateral ventricle. C: Image from second iMRI study, performed after further resection, demonstrating complete removal of the lesion.

\section{Group 3: Other Histological Entities}

This heterogeneous group included 30 patients with different pathologies (Table 1). The most frequent histological diagnoses were ependymoma (3 WHO Grade II cases and 1 WHO Grade III case), ganglioglioma (3 cases) (Fig. 3), and cortical dysplasia (3 cases). For these last 2 pathological entities, the main symptom was epilepsy. In order to obtain optimal influence on seizures, GTR is an important goal, and it was intended in all but 2 cases. The preoperative planned goal (or better) was reached in all surgeries, using 1 iMRI study in 25 cases and 2 iMRI studies in 5 cases.

The 5 operations (17\%) in which resection was continued after the first iMRI study were performed for treatment of cortical dysplasia in 2 cases and for resection of a ganglioglioma, a germinoma, and a neurocytoma in the other 3 cases ( 1 case each). In the 2 cases in which GTR was not intended, PR was planned, but 2 iMRI studies were performed to maximize the resection, and STR was achieved; in both cases the surgeon requested the first iMRI study in order to identify the possible tumor remnant and its relation with eloquent structures. Without this tool the surgery would have been terminated. In the remaining 3 patients (with cortical dysplasia, ganglioglioma, and germinoma), the first iMRI demonstrated residual lesion, which was then removed, and the removal was confirmed by the second iMRI study. In these cases the residual tumor was unexpected, and before the imaging the surgeon described the degree of resection as total removal. The mean total time for the preoperative MRI, including positioning, coil setting, and draping, was $27 \pm$ 5 minutes. The corresponding mean total time for iMRI was $34 \pm 6$ minutes.

Four of the patients with ganglioglioma or cortical dysplasia experienced postoperative resolution of epileptic seizures and 2 showed improvement. Also, in this group there were no intraoperative complications. Postoperatively, 1 patient experienced urinary tract infection and 2 had CSF leakage, which was treated conservatively. In 3 cases there was transient worsening of the patients' neurological status after the operation. The mean total time for the preoperative MRI, including positioning, coil setting, and draping, was $40 \pm 5$ minutes. The corresponding mean total time for iMRI was $43 \pm 6$ minutes.

\section{Discussion}

Previous studies have validated the usefulness of intraoperative imaging in the treatment of intracranial tumors in adults. This tool appears to be safe and effective to increase the extent of resection in the treatment of gliomas, pituitary adenomas, and craniopharyngiomas. ${ }^{10,15,16,18}$ Recently, the German Study Group for iMRI published a retrospective study about iMRI-guided surgery for lowgrade gliomas in adults, observing that high-field iMRI was significantly associated with GTR. ${ }^{6}$ There are few studies about the effectiveness of iMRI for the treatment of intracranial tumors in children or, more important, its safety. ${ }^{1,21}$

In the present study we divided our pediatric population treated with iMRI into patients with sellar region tumors, those with gliomas (supra- and infratentorial), and those with other entities.

Studies of the iMRI-assisted treatment of sellar region tumors in adults have shown that the GTR rate for pituitary adenomas improved significantly ${ }^{3,16}$ due to the visualization of even small tumor remnants. Intraoperative imaging has also been used for craniopharyngioma surgery, providing good early prediction of total removal and facilitating further resection of remnants. ${ }^{3}$ In our pediatric population, we performed 22 craniopharyngioma surgeries in 15 patients. We found that in 5 procedures
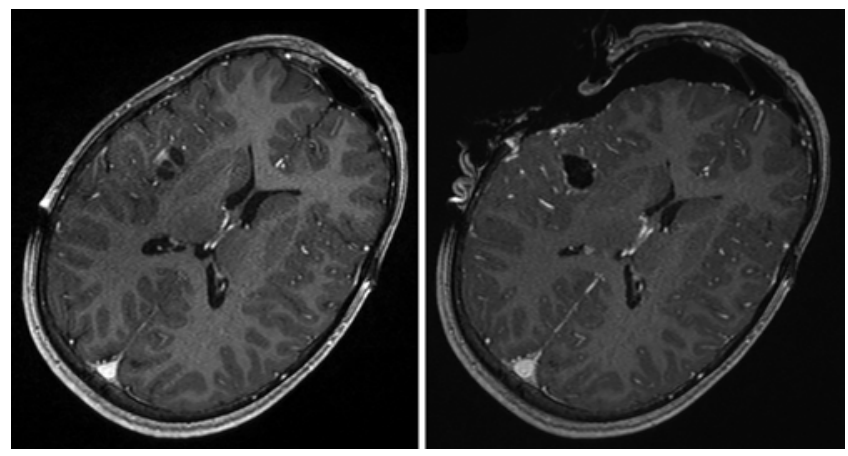

FIG. 3. Axial T1-weighted contrast-enhanced MR images obtained in an 11-year-old child affected by a right insular ganglioglioma. Left: Preoperative image. Right: Intraoperative image demonstrating complete removal of the lesion. 
more than 1 iMRI imaging study was performed, and in 4 of these cases it led to the visualization of tumor remnant and its removal, allowing for complete resection. Thus in $4(18 \%)$ of 22 procedures iMRI identified residual tumor, which led to its complete removal. These data are in accordance with the study involving adults conducted by Hofmann et al. ${ }^{10}$ On the other hand, as already stated in the cited literature, the extended resection of tumor remnants visualized with iMRI does not exclude recurrence, originating mainly from tiny capsule remnants that cannot be detected by the currently available iMRI resolution.

Skull base development is complex and asymmetrical: pneumatization of the sphenoid sinus has different patterns and rate of progress. ${ }^{2}$ An incompletely pneumatized sphenoid sinus can pose greater difficulties during a transsphenoidal route in young children, as occurred in one of our cases. The use of iMRI paired with the navigation system may be of help for better intraoperative orientation during this approach.

To evaluate the real utility of iMRI, it is important to consider the intraoperative impression of the surgeon and his or her assessment before performing the iMRI imaging session. As previously mentioned, the imaging procedure is requested in 3 possible situations for 3 different purposes: 1) to confirm the impression that a surgical goal (such as GTR) has been achieved, 2) to identify and quantify a suspected tumor remnant, and 3) to update the navigation system such as in case of severe brain shift (with new DTI sequences for depiction of fiber bundles) or anatomical anomaly (partially pneumatized sphenoid sinus).

In 3 cases of supratentorial gliomas and 1 case of infratentorial glioma in which a GTR was planned, the first iMRI study showed residual tumor even though the surgeon had the impression of complete removal. Thus in 4 (27\%) of 15 cases, after iMRI depiction of residual tumor the surgeon continued the procedure with updated navigation to obtain GTR, reaching the preoperatively planned goal and avoiding the necessity for reoperation.

Other important data comes from the analysis of the 10 glioma cases in which only a partial resection was intended: iMRI allowed for extension of the planned removal from partial to subtotal in $50 \%$ of the cases. This result is due not only to the detection and localization of the residual tumor, but also to the improved "feeling of safety" given to the surgeon by the visualization of the relation of remnant tumor and functionally critical structures such as the corticospinal tract and the ability to update the navigation system. In fact, in these cases the surgeon stated that there was residual tumor before it was confirmed by iMRI, but its volume and relation to eloquent structures were uncertain; thus, the surgery would have been stopped without iMRI.

Similarly, in the category of other histological entities (Group 3) 5 surgeries were continued after the first iMRI. In 3 cases GTR could be achieved due to the visualization of residual lesion that was not expected by the surgeon (the removal was judged complete before the imaging); in 2 cases planned for PR, the resection could be extended to STR after imaging (the surgeon would otherwise not have continued the operation). In this last group of patients,
iMRI was particularly useful for lesions that were difficult to differentiate microscopically from normal tissue.

The data obtained in our pediatric population confirm the results of the studies in adult patients about the increased extent of resection using iMRI. ${ }^{6,15,18}$ Other studies about the use of intraoperative imaging during resection of pediatric brain tumors confirm the utility of this tool. Our data on further tumor resection using iMRI are confirmed by the recent literature. Yousaf et al. ${ }^{21}$ used iMRI in 79 procedures for the treatment of intracranial tumors in 73 children. In $26 \%$ of the 47 procedures in which a complete resection was intended, the identification and removal of residual lesion was possible after the first iMRI scan. In $41 \%$ of the 32 procedures in which partial removal was planned, the resection could be extended. Similar results were obtained in the series published by Choudhri et al., ${ }^{5}$ which comprised patients affected mainly by low-grade gliomas and medulloblastomas: additional tumor was resected in $21 \%$ of the cases after scanning. Moreover, in $50 \%$ of the 40 cases in which debulking was planned, a near-total resection could be achieved, and in 5\%, GTR was achieved. The same authors showed a significant reduction in the early reoperation rate from $8 \%$ to $1 \%$ with the introduction of iMRI. ${ }^{1,5}$ Reduction of reoperations was also reported by Avula et al., ${ }^{1}$ who reported a reduction to $0 \%$ (for repeat operations within 6 months of the index procedure) in a population of 36 patients treated with the aid of iMRI. These data can be corroborated by our study, in which no patient underwent reoperation during the early postoperative period. In the cited literature all the authors could confirm the benefits offered by iMRI with respect to maximizing intracranial tumor resection and improving navigation accuracy, compensating for brain shift. ${ }^{21}$ In our experience we observed that patients with low-grade gliomas, especially pilocytic astrocytomas, could particularly benefit from iMRI because of the difficulty of differentiating these lesions from normal tissue during surgery; in such histological entities a complete removal is important because it may be curative. In general, iMRI can be expected to be helpful in the resection of lesions located close to functional areas (motor and/or speech) and major fiber bundles (i.e., corticospinal tract) due to the possibility of navigation update and shift compensation. Solid and firmly capsulated sellar region tumors can also benefit from iMRI due to the visualization of capsule remnant.

In our series, there was no intra- or postoperative complication related to the use of iMRI. In the case of epidural hematoma, the intraoperative imaging allowed its visualization and the blood could be immediately removed. The prolongation of surgical time due to preoperative and intraoperative imaging did not influence the results of the surgery.

Performing preoperative imaging in the iMRI environment allows the patient to avoid an additional sedation the day before surgery to obtain navigation sequences and/ or updated images. Moreover, the images obtained in the iMRI environment are obtained after the patient's head is positioned for the surgery, simplifying surgical planning and improving comparability with the intraoperative imaging. 


\section{Technical Limits of iMRI}

The limitations of iMRI in pediatric patients in our setup are mostly due to the configuration of the surgical table. Our table does not include sections that can be adjusted separately, so surgical positioning is limited to prone and supine positions. The sitting position, which we mostly use for posterior fossa lesions, is not possible with this configuration. The pin fixation headrest-coil unit in the theater setup is composed of two half-cylinders placed superior and inferior to the patient's head and can only be partially rotated. This unit is designed to accommodate adults' heads, and its use in pediatric patients, particularly very young ones, may lead to additional challenges. Padding may be needed to adjust the headrest, and this may lead to positional instability during the procedure. We did not have any intraoperative complications due to the above-mentioned configuration, but the prone position proved to be the most difficult and time consuming to achieve; in fact, our data show that the procedures performed for treatment of infratentorial gliomas required the longest preoperative time (mean $49 \pm 5$ minutes). Moreover, in the prone position head flexion was partially limited by the fixed pin pattern. Other publications have also reported difficulties with pin fixation in infants ${ }^{4}$ and adults. ${ }^{8}$ For these reasons, new headrest systems, such as an MRI-compatible horseshoe headrest for infants, are highly desirable.

\section{Conclusions}

The use of iMRI in the pediatric population proved to be safe in this study. In addition, it was effective in increasing the extent of tumor resection, especially in patients affected by low-grade gliomas and craniopharyngiomas. There were no intraoperative complications due to the use of this tool, and the prolonged surgical time was balanced by the exclusion of preoperative and early postoperative MRI, which reduces stress for younger children, in particular, and avoids the potential need for additional sedation. The most prominent disadvantage of high-field iMRI in our series of cases was the limitation in terms of operative positioning due to the configuration of the surgical table.

\section{Acknowledgments}

We would like to thank our radiographers-Mr. L. Hebing, F. Mühler, Ms. U. Kabelitz, and Ms. D. Neumann-for performing the iMRI.

\section{References}

1. Avula S, Pettorini B, Abernethy L, Pizer B, Williams D, Mallucci C: High field strength magnetic resonance imaging in paediatric brain tumour surgery-its role in prevention of early repeat resections. Childs Nerv Syst 29:1843-1850, 2013

2. Banu MA, Guerrero-Maldonado A, McCrea HJ, GarciaNavarro V, Souweidane MM, Anand VK, et al: Impact of skull base development on endonasal endoscopic surgical corridors. J Neurosurg Pediatr 13:155-169, 2014

3. Berkmann S, Schlaffer S, Nimsky C, Fahlbusch R, Buchfelder M: Intraoperative high-field MRI for transsphenoidal reoperations of nonfunctioning pituitary adenoma. J Neurosurg 121:1166-1175, 2014

4. Boop FA, Bate B, Choudhri AF, Burkholder B, Klimo P Jr: Preliminary experience with an intraoperative MRIcompatible infant headholder: technical note. J Neurosurg Pediatr 15:539-543, 2015

5. Choudhri AF, Klimo P Jr, Auschwitz TS, Whitehead MT, Boop FA: 3T intraoperative MRI for management of pediatric CNS neoplasms. AJNR Am J Neuroradiol 35:2382-2387, 2014

6. Coburger J, Merkel A, Scherer M, Schwartz F, Gessler F, Roder C, et al: Low-grade glioma surgery in intraoperative magnetic resonance imaging: results of a multicenter retrospective assessment of the German Study Group for Intraoperative Magnetic Resonance Imaging. Neurosurgery 78:775-786, 2016

7. Gerganov VM, Giordano M, Fahlbusch R, Samii M, Samii $\mathrm{A}$ : Tumor resection control using intraoperative magnetic resonance imaging, in Hayat MA (ed): Tumors of the Central Nervous System, Volume 4. Brain Tumors (Part 2). Dordrecht: Springer, 2012, pp 167-173

8. Giordano M, Gerganov VM, Metwali H, Fahlbusch R, Samii A, Samii M, et al: Feasibility of cervical intramedullary diffuse glioma resection using intraoperative magnetic resonance imaging. Neurosurg Rev [epub ahead of print], 2013

9. Hall WA, Martin AJ, Liu H, Pozza CH, Casey SO, Michel E, et al: High-field strength interventional magnetic resonance imaging for pediatric neurosurgery. Pediatr Neurosurg 29:253-259, 1998

10. Hofmann BM, Nimsky C, Fahlbusch R: Benefit of 1.5-T intraoperative MR imaging in the surgical treatment of craniopharyngiomas. Acta Neurochir (Wien) 153:13771390, 2011

11. Knauth M, Wirtz CR, Tronnier VM, Aras N, Kunze S, Sartor $\mathrm{K}$ : Intraoperative MR imaging increases the extent of tumor resection in patients with high-grade gliomas. AJNR Am J Neuroradiol 20:1642-1646, 1999

12. Lam CH, Hall WA, Truwit CL, Liu H: Intra-operative MRIguided approaches to the pediatric posterior fossa tumors. Pediatr Neurosurg 34:295-300, 2001

13. Levy R, Cox RG, Hader WJ, Myles T, Sutherland GR, Hamilton MG: Application of intraoperative high-field magnetic resonance imaging in pediatric neurosurgery. $\mathbf{J}$ Neurosurg Pediatr 4:467-474, 2009

14. Martin C, Alexander E III, Wong T, Schwartz R, Jolesz F, Black PM: Surgical treatment of low-grade gliomas in the intraoperative magnetic resonance imager. Neurosurg Focus 4(4):e8, 1998

15. Nimsky C, Fujita A, Ganslandt O, Von Keller B, Fahlbusch R: Volumetric assessment of glioma removal by intraoperative high-field magnetic resonance imaging. Neurosurgery 55:358-371, 2004

16. Nimsky C, von Keller B, Ganslandt O, Fahlbusch R: Intraoperative high-field magnetic resonance imaging in transsphenoidal surgery of hormonally inactive pituitary macroadenomas. Neurosurgery 59:105-114, 2006

17. Rodríguez D, Cheung MC, Housri N, Quinones-Hinojosa A, Camphausen K, Koniaris LG: Outcomes of malignant CNS ependymomas: an examination of 2408 cases through the Surveillance, Epidemiology, and End Results (SEER) database (1973-2005). J Surg Res 156:340-351, 2009

18. Senft C, Bink A, Franz K, Vatter H, Gasser T, Seifert V: Intraoperative MRI guidance and extent of resection in glioma surgery: a randomised, controlled trial. Lancet Oncol 12:997-1003, 2011

19. Steinmeier R, Fahlbusch R, Ganslandt O, Nimsky C, Buchfelder M, Kaus M, et al: Intraoperative magnetic resonance imaging with the Magnetom Open scanner: 
concepts, neurosurgical indications, and procedures: a preliminary report. Neurosurgery 43:739-748, 1998

20. Wisoff JH, Boyett JM, Berger MS, Brant C, Li H, Yates AJ, et al: Current neurosurgical management and the impact of the extent of resection in the treatment of malignant gliomas of childhood: a report of the Children's Cancer Group trial no. CCG-945. J Neurosurg 89:52-59, 1998

21. Yousaf J, Avula S, Abernethy LJ, Mallucci CL: Importance of intraoperative magnetic resonance imaging for pediatric brain tumor surgery. Surg Neurol Int 3 (Suppl 2):S65-S72, 2012

\section{Disclosures}

The authors report no conflict of interest concerning the materi- als or methods used in this study or the findings specified in this paper.

\section{Author Contributions}

Conception and design: Giordano, A Samii, Fahlbusch, Di Rocco. Acquisition of data: Giordano, Lawson McLean. Analysis and interpretation of data: Giordano. Drafting the article: Giordano. Critically revising the article: Giordano, A Samii, Fahlbusch, Di Rocco. Reviewed submitted version of manuscript: A Samii. Study supervision: Bertalanffy, M Samii, Di Rocco.

\section{Correspondence}

Mario Giordano, International Neuroscience Institute-Hannover, Rudolf Pichlmayr Str. 4, Hannover 30625, Germany. email: mario.giordano@alice.it. 